\title{
Selective, Laser-Induced Etching of Fused Silica at High Scan-Speeds Using KOH
}

\author{
Martin HERMANS ${ }^{* 1,2}$, Jens GOTTMANN ${ }^{* 1,2}$ and Frank RIEDEL ${ }^{* 3}$ \\ ${ }^{* 1}$ RWTH Aachen University, Chair for Lasertechnology LLT, Steinbachstraße 15, 52074 Aachen, \\ Germany \\ E-mail:martin.hermans@llt.rwth-aachen.de \\ *2 LightFab UG, Steinbachstraße 15, 52074 Aachen, Germany \\ ${ }^{*}$ Fraunhofer ILT, Steinbachstraße 15, 52074 Aachen, Germany
}

\begin{abstract}
Selective, laser-induced etching (SLE) is a process which offers the possibility of machining hollow volumes into transparent materials with a huge freedom of geometry in 3D. Every 3D structure consists of single lines of laser-induced modifications. The knowledge of selectivity for etching of these single lines of modification is crucial to identify stable process windows for the machining of completely integrated, complex 3D structures. The selectivity of laser-induced etching of single line modifications is investigated in this study for a variation of repetition rate, pulse duration, pulse energy and feed rate for etching with $\mathrm{KOH}$.
\end{abstract}

DOI: 10.2961/jlmn.2014.02.0009

Keywords: Ultrashort, hybrid, etching, $\mathrm{KOH}$, fused silica, scanning, high-speed, laser, machining, $3 \mathrm{D}$, microfluidics

\section{Introduction}

Focusing ultrashort pulsed laser radiation with a NA $>0.2$ leads to focal intensities in the order of $I>10^{12} \mathrm{~W} / \mathrm{cm}^{2}$ at pulse energies in the order of hundreds of nanojoule. At these intensities non-linear photoionization occurs in the vicinity of the focus when it is positioned within the volume of a dielectric transparent to the wavelength of the laser radiation. The generation of free electrons by nonlinear absorption enables further absorption of radiation by Bremsstrahlung absorption leading to avalanche-like increase of the number of free electrons [1]. The energy of the electron system excited like that is partially transferred to the atoms via electron-phonon coupling and leads to heating of the material up to temperature differences in the order of several $1000 \mathrm{~K}$ depending on the material and parameters of irradiation [2]. Moving the focal spot with feed rates $>100 \mathrm{~mm} / \mathrm{s}$ at pulse repetition rates $>500 \mathrm{kHz}$ through the material leads to rapid quenching of the material and thus permanently freezing in an high temperature, high pressure state of the material. By moving the focal spot through the material arbitrarily in $3 \mathrm{D}$, a continuous lineshaped modification is formed. In case the modified material has somewhere contact to the outer side of the sample, putting the sample in a suitable wet etching agent as a second process step results in the solution of the modified material at a higher rate than the non-modified material. The ratio between the etching rates of modified and nonmodified material is called selectivity and is equivalent to the possible maximum aspect ratio of machinable structures. This two-step process of modifying material with laser radiation and removing mainly the modified material with a subsequent step of wet etching is called Selective Laser-induced Etching (SLE). This process offers unique features for machining arbitrary hollow volumes within transparent materials like crystals or glasses with precisions in the order of $1 \mu \mathrm{m}$ by stacking single line-shaped modifications to get the desired shape.

Marcinkevicius et al.was the first group to report about using SLE for micro fabrication of 3D microfluidic channels in fused silica with $\mathrm{HF}$ as etching agent [3]. For $2.5 \%$ HF etching selectivities up to $\sim 100$ and an etching rate of $70 \mu \mathrm{m} / \mathrm{h}$ at a feed rate of $30 \mathrm{~mm} / \mathrm{s}$ can be achieved [4].

For selective etching in fused silica HF can be substituted as etching agent with $\mathrm{KOH}$ with the advantage of a reduced harmfulness to the human body and a constant selectivity in dependence of the length of the evolving micro channel achieving selectivities up to 350 [5]. This is why the authors of the study at hand focused their investigations on etching the laser-induced line modifications with $\mathrm{KOH}$.

Applications for $S L E$ range from the simple creation of precise cuts to the fabrication of complete already-mounted micro-mechanical systems and microfluidic elements [610] (Figure 1). Speeding up the process is necessary for establishing $S L E$ as an economically worthwhile production

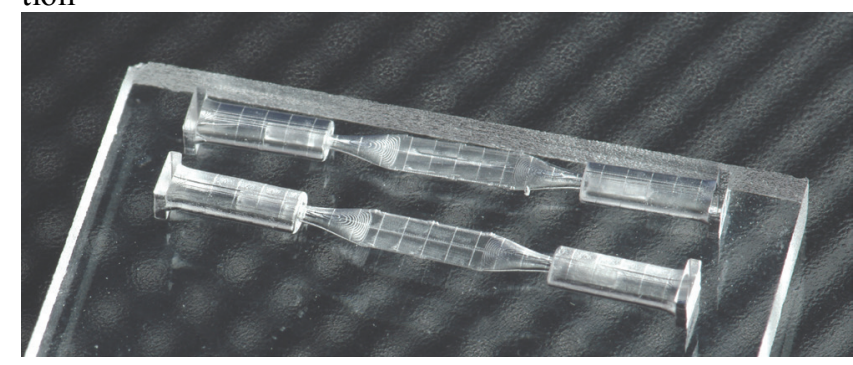


Figure 1 Micro-fluidic chip with built-in side connectors for attachment of flexible tube for automated cell diagnostics fabricated inside fused silica by SLE; length $15 \mathrm{~mm}$, height $2 \mathrm{~mm}$

process. This can be achieved by the development of faster scanning systems for spot sizes in the micrometer range and has already been reported up to feed rates in the order of $10 \mathrm{~m} / \mathrm{s}$ within fused silica [11]. In order to overcome process limitations like crack formation it is crucial to have knowledge about the dependency of etching selectivity from pulse repetition rate, pulse energy and feed rate for single line-shaped modifications before stacking singe lines to complex 3D structures.

While for feed rates in the order of $1 \mathrm{~mm} / \mathrm{s}$ the aforementioned dependencies have been reported for fused silica using $\mathrm{KOH}$ as etching agent, this data is not available for feed rates up to $200 \mathrm{~mm} / \mathrm{s}$. By the results of this work this gap is intended to be closed.

\section{Experimental Setup \& Procedure}

Lines of laser induced modifications are inscribed $200 \mu \mathrm{m}$ beneath the surface of flat test pieces of fused silica (Suprasil1, Heraeus GmbH; Hanau, Germany). The laser source used for the experiments is a FCPA laser (Satsuma, Amplitude Systèmes; Bordeaux, France) providing pulsed laser radiation at a wavelength of $1030 \mathrm{~nm}$ with pulse durations ranging from 300 to 3000 fs with a maximum pulse energy of $20 \mu \mathrm{J}$ at $500 \mathrm{kHz}$ and repetition rates ranging from 0 to $17 \mathrm{MHz}$. Laser radiation is focused by a 20x microscope objective with a numerical aperture of 0.45 (LCPLAN N 20x/0.45 IR, Olympus Europa GmbH; Hamburg, Germany) equipped with a collar for correction of spherical aberrations. The spherical aberrations of a noncollimated beam at a plane surface are compensated according to the depth of modification. Measurement of the focal spot diameter $2 w_{0}$ with a $40 \mathrm{x} / 0.8$ microscope objective in combination with a linearized CMOS camera (BladeCam XHR, Data Ray Inc.; Bellavista, USA) yields $2 w_{0}=2.2 \mu \mathrm{m}$ with a resolution of the system better than $1.7 \mu \mathrm{m}$ (Figure 2).

\begin{tabular}{||lr|}
\hline Clip[a] & $13.5 \%$ \\
\hline 4xSigma[b] & Camera 1: Della = 6.9 um PIxeII = $58048[88.6 \%]$ \\
\hline \hline Running \#1 xBlade. 2 \\
\hline \hline Major
\end{tabular}

Figure 2 Measurement of focal spot diameter with custom made setup; resolution $<1.7 \mu \mathrm{m}$

Polarization of the laser radiation is linear and chosen to be perpendicular to the feed rate vector due to the fact that this orientation of polarization yields the highest selectivities [5,6]. The fused silica samples are mounted and aligned on a xy-system of air bearing stages (ABL 10100 and ABL 20030, Aerotech Inc.; Pittsburgh, USA). For irradiation the parameters pulse energy $E_{p}$, repetition rate $f_{\text {rep }}$, feed rate $v_{x y}$, and pulse duration $t_{p}$ are varied according to table 1. Pulse energy is measured below the microscope objective without sample. Modifications are inscribed 3 times back and forth respectively resulting in 6 lines of modification for each parameter combination with a distance of $100 \mu \mathrm{m}$ between each line.

Table 1 Parameter variation for irradiation

\begin{tabular}{lll}
\hline Parameter & Variation & Steps/Value \\
\hline Pulse energy $E_{p}$ & $100-1000 \mathrm{~nJ}$ & $100 \mathrm{~nJ}$ \\
Repetition rate $f_{r e p}$ & $250-4000 \mathrm{kHz}$ & $250 \mathrm{kHz}$ \\
Feed rate $v_{x y}$ & $50-200 \mathrm{~mm} / \mathrm{s}$ & $50 \mathrm{~mm} / \mathrm{s}$ \\
Pulse duration $t_{p}$ & $300-1000 \mathrm{fs}$ & $100 \mathrm{fs}$ \\
\hline
\end{tabular}

After irradiation and modification the samples are grinded in the plane spanned from the feed rate vector during line modification in order to make sure that the modified material has contact to the outer side of the sample.

The samples are placed into a wet-etching bath of potassium hydroxide $(\mathrm{KOH})$ with a concentration of $8 \mathrm{~mol} / \mathrm{l}$ at a temperature of $85^{\circ} \mathrm{C}$ for 6 hours.

After wet-etching the developed in-volume microchannels are being investigated by transmitted light microscopy with polarization contrast, the length of the channel $l$ is measured and subsequently the rate of selective etching $r_{s}$ is calculated. The unetched modification appears bright due to birefringence resulting from nanogratings (NG) and/or stress induced birefringence. The etched micro channel appears black (Figure 3) with no birefringence left behind.

In order to determine the selectivity $S$ of the etching process, the ratio of etch rates is calculated according to

$$
S=\frac{r_{s}+r_{0}}{r_{0}}
$$

Where $r_{0}$ denotes the etch rate of non-modified fused silica which is measured to be $0.21 \pm 0.015 \mu \mathrm{m} / \mathrm{h}$ at a temperature of $85^{\circ} \mathrm{C}$ and a concentration of $8 \mathrm{~mol} / 1$ being in good agreement with an etching rate of $0.25 \mu \mathrm{m} / \mathrm{h}$ at $80^{\circ} \mathrm{C}$ and $10 \mathrm{~mol} / \mathrm{l}$ as reported in [5].

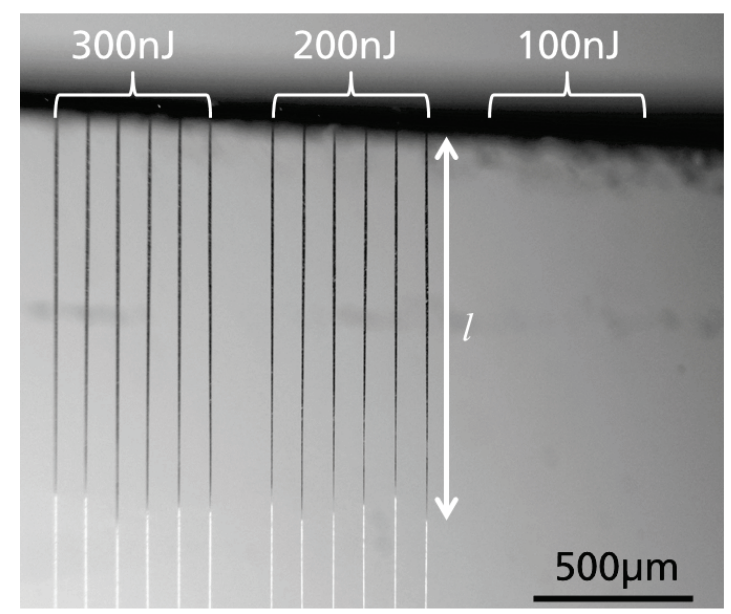

Figure 3 Micro graph with polarization contrast of modified and etched sample, micro channels with length $l$ appear black, unetched modification appear bright due to birefringence; $f_{\text {rep }}=750 \mathrm{kHz}, t_{p}=800 \mathrm{fs}, v_{x y}=100 \mathrm{~mm} / \mathrm{s}$ (top view) 


\section{Results \& Discussion}

The general dependencies of selectivity on the parameters varied for the study at hand are discussed and are shown exemplarily for chosen parameters. The largest selectivity found in this study is $S \sim 1400$ together with a selective etching rate of $r_{s} \sim 290 \mu \mathrm{m} / \mathrm{h}$.

For all the parameters investigated the selectivity depends similarly on the pulse energy applied. For a pulse energy $E_{p}=100 \mathrm{~nJ}$ no visible line of modification can be detected and no micro channels have developed after etching (Figure 3). For $200 \mathrm{~nJ}$ a visible modification along with an etched micro channel can be observed corresponding to a threshold fluence for modification of $\sim 5 \mathrm{~J} / \mathrm{cm}^{2}$.

Two process windows for selective etching are found. The highest selectivity for micro channels is observed along with straight and smooth sidewalls pulse energies between 200 and $500 \mathrm{~nJ}$ within the range of all repetition rates, feed rates and pulse durations investigated. For pulse durations $t_{p}>400$ fs the maximum selectivity does not depend on pulse duration in terms of measurement accuracy (Figure 4) within this first process window.

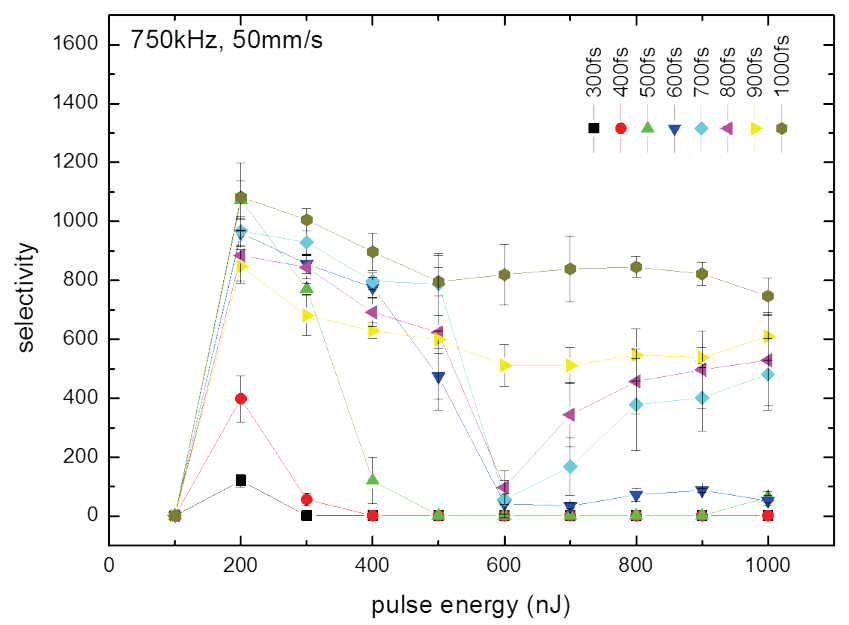

Figure 4 Selectivity vs. pulse energy; $f_{\text {rep }}=750 \mathrm{kHz}, 50 \mathrm{~mm} / \mathrm{s}$

The second process window for selective etching is found for pulse energies $E_{p}>700 \mathrm{~nJ}$. The micro channels feature irregular sidewalls presumably originating from micro cracks widened by the etching process (Figure 5 inlay left). For pulse durations $t_{p}<900$ fs selectivity between the two process windows drops. Furthermore for $t_{p}<500$ fs and $v_{x y}<100 \mathrm{~mm} / \mathrm{s}$ the second process window does not exist. One difference between those two process windows is revealed by a more detailed view on the unetched modifications. The structure of the line modification (Figure 6) for $600 \mathrm{~nJ}$ pulse energy features irregularities larger than $2 \mu \mathrm{m}$ which are formed by periodically appearing bubbles or maybe voids surrounded by an in transmission light microscopy brighter appearing material. This brighter appearing material probably consists of molten and resolidified material (Figure 6).

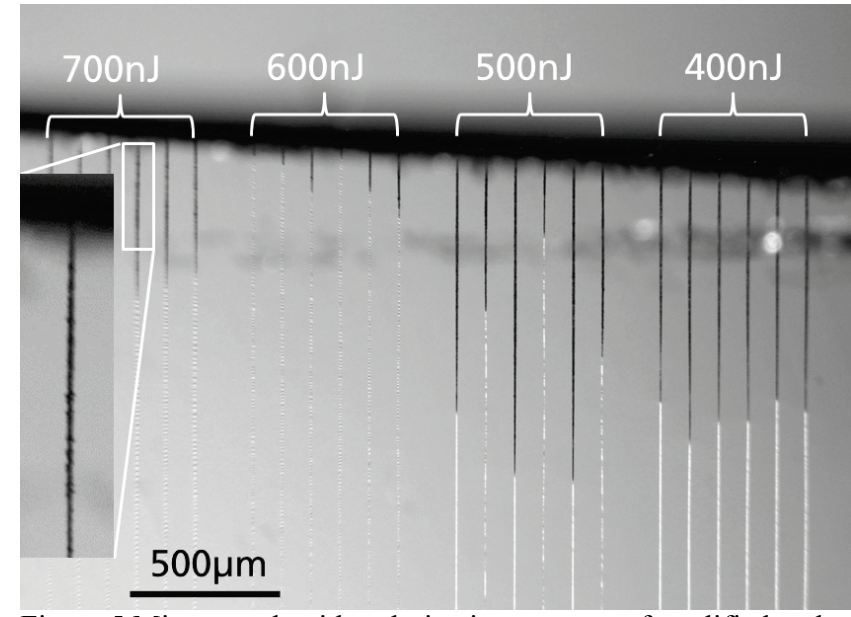

Figure 5 Micro graph with polarization contrast of modified and etched sample, micro channels appear black, unetched modification appear bright due to birefringence; for a pulse energy $E_{p}=600 \mathrm{~nJ}$ a decrease in selectivity is observable; $f_{\text {rep }}=750 \mathrm{kHz}$, $t_{p}=800 \mathrm{fs}, v_{x y}=50 \mathrm{~mm} / \mathrm{s}$ (top view)

For $E_{p}=500 \mathrm{~nJ}$ these irregularities can be observed only for one direction of the feed rate indicating a defined threshold for the development of the irregularities during modification (Figure 6). A different energy deposition for the forth and back direction could be attributed to a quill writing effect resulting from a tilted pulse front as reported in [12].

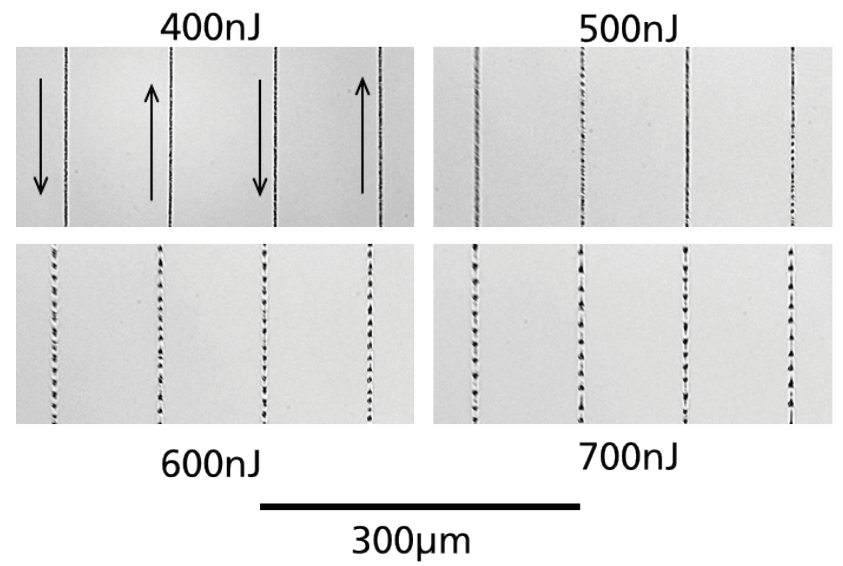

Figure 6 Micro graph in transmitted light microscopy of involume modification at different pulse energies applied (top view); back and forth direction is indicated by arrows; $f_{\text {rep }}=750 \mathrm{kHz}, t_{p}=800 \mathrm{fs}, v_{x y}=50 \mathrm{~mm} / \mathrm{s}$

Furthermore appearing irregularities and probably molten and resolidified material coincide with a lower selectivity observed after wet-etching (Figure 5).

For repetition rates $f_{\text {rep }}>1000 \mathrm{kHz}$ no selective etching is observed at all. The line modifications within this regime feature molten material periodically interrupted by bubbles as reported in [14].

At higher feed rates the first process window for pulse durations $t_{p}<600 \mathrm{fs}$ is larger (Figure 7), i.e. for a wider range of pulse energies a selectivity $S>800$ is observed. The second process window exists for all pulse durations investigated, still separated by a dip in selectivity from the first process window for $t_{p}<600$ fs. Corbari et al. also found a dip in selectivity at interim fluences for femtosecond pulses 
while for picosecond pulses they observed an almost constant selectivity [13].

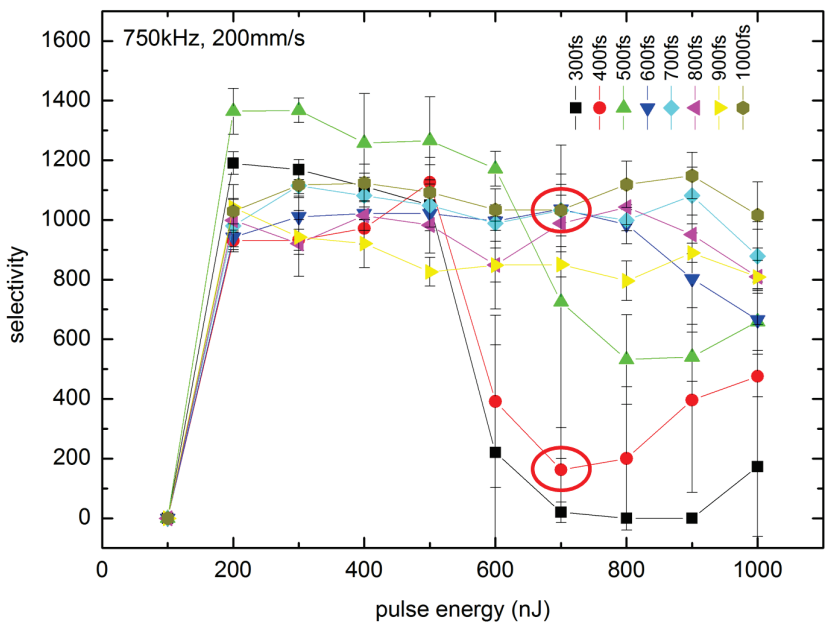

Figure 7 Selectivity vs. pulse energy; $f_{\text {rep }}=750 \mathrm{kHz}, 200 \mathrm{~mm} / \mathrm{s}$

Comparison of two unetched line modifications at different pulse durations one exhibiting the dip in selectivity one not (red circles in Figure 7) by means of polarization contrast microscopy reveals differences in terms of birefringence. For a pulse duration of $t_{p}=400 \mathrm{fs}$ the observed birefringence does not depend on the angle between line modification and the polarization of the light for illumination (Figure 8 top row) and is comparably weak. For a pulse duration of $t_{p}=1 \mathrm{ps}$ the birefringence exhibits an anisotropy in relation to the orientation of the polarization of the illumination light. Rotating the sample by an angle of $45^{\circ}$ leads to a clear drop in intensity of the observed light (Figure 8 bottom row). Rotating the sample further by an angle of $45^{\circ}$ the maximum intensity is found again. Furthermore the observed birefringence is stronger than for $t_{p}=400 \mathrm{fs}$.

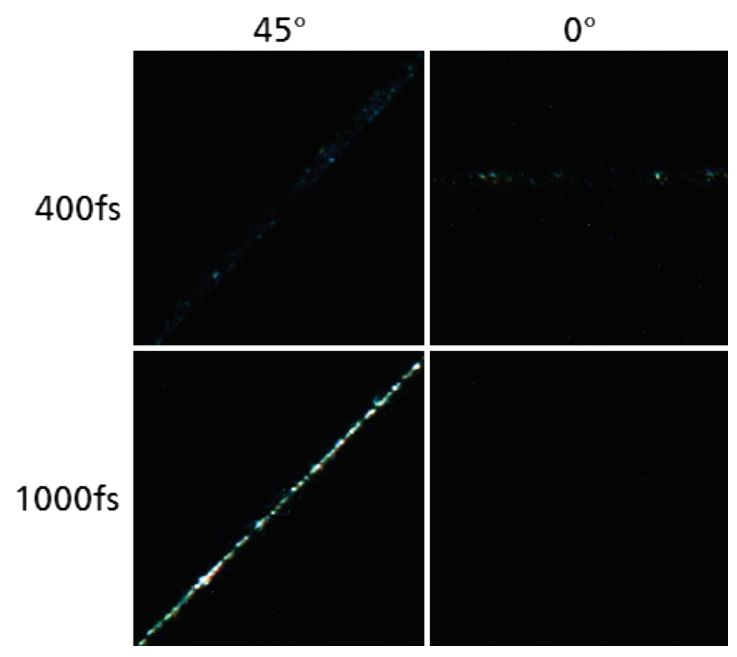

Figure 8 Micro graph of unetched line modifications for two different pulse durations (top: $400 \mathrm{fs}$, bottom: $1 \mathrm{ps}$ ) in polarization contrast at two different angles in relation to the orientation of the first polarizer, micro graphs taken under same exposure time and ISO number; $E_{p}=700 \mathrm{~nJ}, f_{\text {rep }}=750 \mathrm{kHz}, v_{x y}=200 \mathrm{~mm} / \mathrm{s}$

According to [15] and [16] the anisotropy in birefringence is attributed to the formation of NG's within the line modification. This leads to the conclusion that at otherwise equal parameters for irradiation the formation of NG's is found for $t_{p}=1000 \mathrm{fs}$ whereas for $t_{p}=400 \mathrm{fs}$ it is not. This implies that the classification introduced by Hnatovsky et al. in [17] has to be extended and modified for pulse durations around $1 \mathrm{ps}$. There is a larger range of pulse energies where NG's are found for 1 ps than for 400 fs (called regime 2 in [17]). The observed higher selective etching rate for a line modification exhibiting NG's furthermore supports the idea that the formation of NG's are at least one driving force for selective etching as expressed in [18]. The temporal formation dynamics of NG's and the possible decomposition of $\mathrm{SiO}_{2}$ into $\mathrm{SiO}$ and $\mathrm{O}^{+}$as suggested by Canning et al. in [19] has to be investigated more intensely in order to explain the presence of NG's for a pulse duration of 1 ps while irradiation with 400 fs does not lead to the formation of NG's.

In order to discuss the differences in selectivity found for $t_{p}=1 \mathrm{ps}$ and $t_{p}=400 \mathrm{fs}$ in dependence of all other parameters varied the results are visualized in terms of the net fluence $F_{n e t}$ as proposed in [13]. There the net fluence is defined as the accumulated deposited energy per area. It can be calculated as the product of the number of pulses per spot $N_{\text {eff }}$ and the fluence $F$ :

$$
F_{n e t}=N_{e f f} \cdot F=\frac{2 w_{0} \cdot f_{r e p}}{v_{x y}} \cdot \frac{E_{p}}{\pi w_{0}^{2}}
$$

For a pulse duration of $t_{p}=400 \mathrm{fs}$ and $f_{\text {rep }}=250-750 \mathrm{kHz}$ a process window exists for net fluences up to $\sim 100$ $150 \mathrm{~J} / \mathrm{cm}^{2}$ (Figure 9).

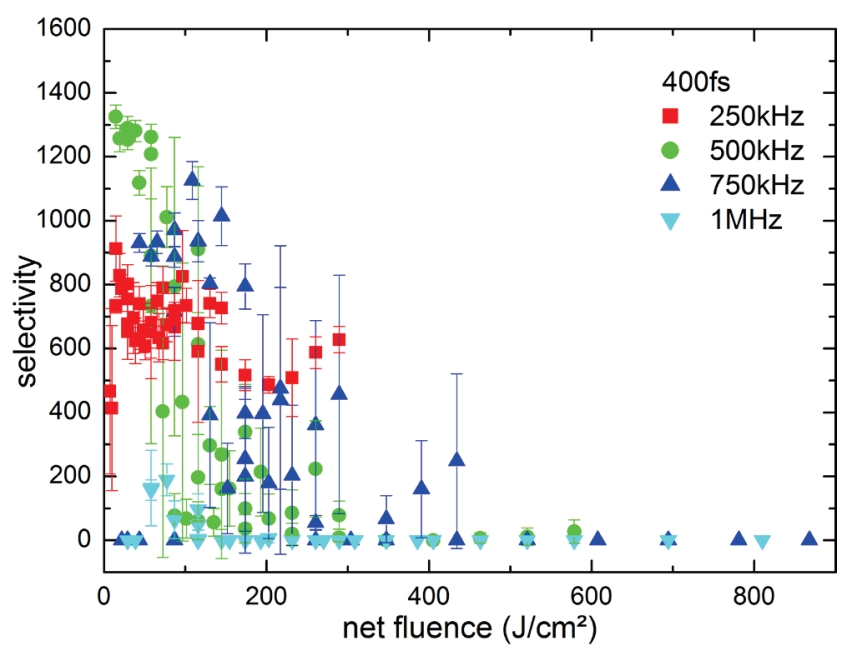

Figure 9 Selectivity vs. net fluence $F_{n e t}$ for the variation of $v_{x y}, f_{\text {rep }}$ and $E_{p}$ investigated at a fixed pulse duration of $t_{p}=400 \mathrm{fs}$.

Higher net fluences lead to formation of channels featuring irregularities at their sidewalls as seen in the inset of Figure 5 also indicated by larger error bars in Figure 9. The maximum selectivity depends on the pulse repetition rate and is highest for $f_{\text {rep }}=500 \mathrm{kHz}$ with $\sim 1300$ and lowest for $f_{\text {rep }}=250 \mathrm{kHz}$ with $\sim 700$. No process window is found for $f_{\text {rep }}=1 \mathrm{MHz}$.

For a pulse duration of $t_{p}=1 \mathrm{ps}$ and $f_{\text {rep }}=250-750 \mathrm{kHz}$ a process window exists for net fluences up to $\sim 800 \mathrm{~J} / \mathrm{cm}^{2}$ (Figure 10). Especially for $750 \mathrm{kHz}$ an almost constant se- 
lectivity is observed over a broad range of net fluences applied.

For $f_{\text {rep }}=1 \mathrm{MHz}$ a process window exists, but is smaller than for the other repetition rates investigated. For net fluences $F_{n e}>200 \mathrm{~J} / \mathrm{cm}^{2}$ heat accumulation starts to play a role.

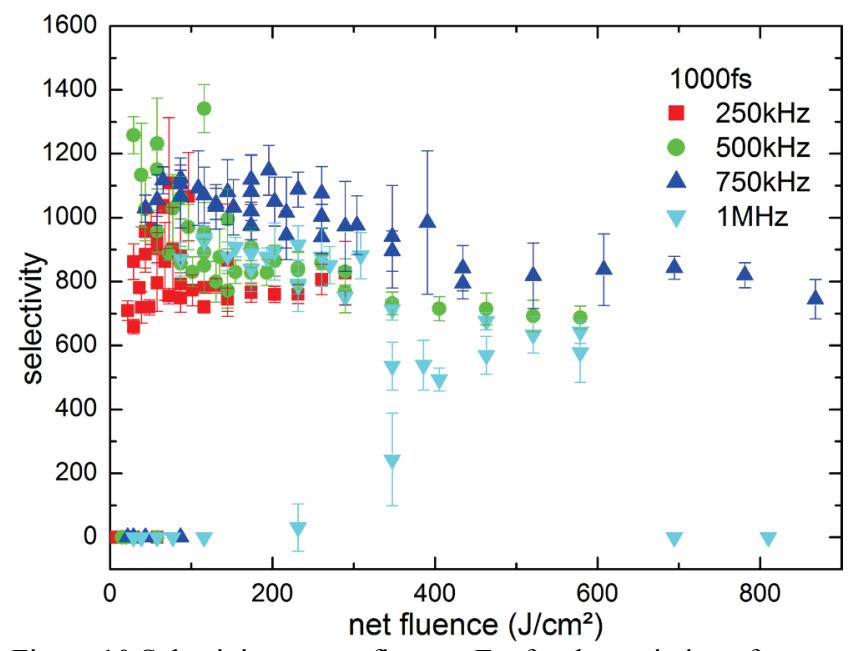

Figure 10 Selectivity vs. net fluence $F_{n e t}$ for the variation of $v_{x y}$, $f_{\text {rep }}$ and $E_{p}$ investigated at a fixed pulse duration of $t_{p}=1 \mathrm{ps}$.

As already stated above several groups have reported that the NG's are on nano-scale periodically alternating layers of pristine $\mathrm{SiO}_{2}$ and $\mathrm{SiO}_{2-x}$ with nano-sized bubbles of $\mathrm{O}_{2}[19]$. The selective etching of fused silica in a solution of $\mathrm{KOH}$ can then be attributed to the chemical reaction of $\mathrm{OH}^{-}$ ions with the silicon-richer material in the NG's [5]. This gives rise to comparison of the selective etching rates in a solution of $\mathrm{KOH}$ found in the present study with those from c-silicon and thermally grown $\mathrm{SiO}_{2}$ reported in literature [20]. The maximum etch rate found within the range of parameters applied for investigation is $290 \pm 7 \mu \mathrm{m} / \mathrm{h}$ which is about a factor of 2-3 higher than the etch rate reported for c-silicon in the $<110>$ and in the $<100>$ plane respectively (Figure 11).

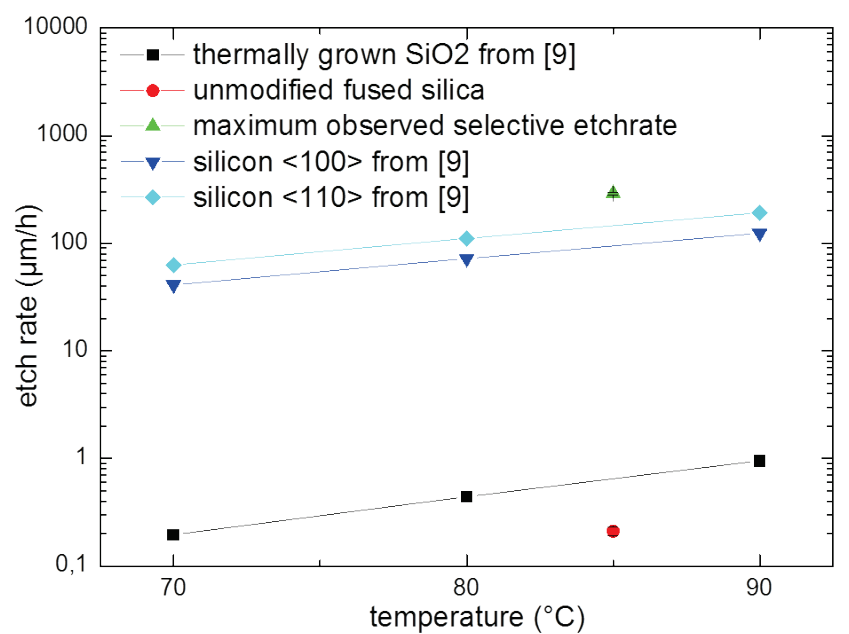

Figure 11 Etch rate vs. temperature for comparison of etch rates in $\mathrm{KOH}$ of thermally grown $\mathrm{SiO}_{2}$, unmodified fused silica as used in the present study, silicon for different orientations and the maximum observed etch rate of a modified line structure
The etch rate of thermally grown $\mathrm{SiO}_{2}$ reported in the literature [20] is $\sim 3$ times higher than the etch rate observed for unmodified fused silica in this study.

The deviation of the etch rates between laser-modified fused silica and c-silicon can be explained by the speculation that, in case there is silicon-rich material within the NG's, it could be in an amorphous state which presumably shows higher etch rates towards $\mathrm{KOH}$ than the c-silicon. To the best knowledge of the authors the etch rate of amorphous silicon towards KOH hasn't been reported yet. Furthermore the nanoporous material offers a higher surface for the chemical reaction so that a higher rate of selective etching can be attributed to this.

\section{Conclusion}

In the present study the dependence of laser-induced selective etching on pulse duration, feed rate, repetition rate and pulse energy for a numerical aperture of $N A=0.45$ is investigated. Process windows for machining fused silica with SLE are identified with maximum selectivities up to $\sim 1400$ and selective etching rates up to $\sim 290 \mu \mathrm{m} / \mathrm{h}$. By the best knowledge of the authors this is the highest observed selectivity and selective etching rate for the SLE process in fused silica. The selective etching rate with $\mathrm{KOH}$ is even higher than for etching with $\sim 2 \% \mathrm{HF}$ reported in $[5,6,18]$. Irradiation with pulse durations $t_{p}<600$ fs lead to a varying width of process window. Wider process windows can be found for $t_{p}>800 \mathrm{fs}$ attributed to a more stable formation of NG's at these pulse durations.

The SLE process is scalable to higher feed rates without sacrificing selectivity within the range of parameters used for irradiation.

The largest selective etch rate found is significantly higher than the one of crystalline silicon under the same etching conditions reported in the literature.

\section{Acknowledgement}

The work presented has been funded by NRW.Transfer Science-to-Business Pre-Seed by the European Union and the local government of Northrhine-Westphalia, Germany. The project has been granted to prepare the spin-off LightFab from the RWTH Aachen University.

The above results were acquired using facilities and devices funded by the Federal State of North-Rhine Westphalia and the European Union within the EFRE-program "Regionale Wettbewerbsfähigkeit und Beschäftigung 20072013" under grant number 290047022

\section{References}

[1] A. Vogel, J. Noack, G. Hüttman, G. Paltauf, "Mechanisms of femtosecond laser nanosurgery of cells and tissues", Appl. Phys. B 81, (2005), 10151047

[2] M. Hermans, J. Gottmann, A. Schiffer, "Insitu diagnostics on fs-laser-induced modifications of glasses for selective etching", Proc. SPIE, 8244, (2012) 
[3]

A. Marcinkeviciius, S. Juodkazis, M. Watanabe, M. Miwa, S. Matsuo, H. Misawa, „Femtosecond laser-assisted three-dimensional microfabrication in silica“, Optics Letters, Vol. 26 No. 5, (2001), pp. $277-279$

[4] S. Rajesh, Y. Bellouard, "Towards fast femtosecond laser micromachining of fused silica: The effect of deposited energy", Optics Express, 18, (2010)

[5] S. Kiyama, S. Matsuo, S. Hashimoto, Y. Morihira, "Examination of Etching Agent and Etching Mechanism on Femtosecond Laser Microfabrication of Channels Inside Vitreous Silica Substrates", J. Phys. Chem. C 113, (2009), 11560-11566

[6] C. Hnatovsky, R.S. Taylor, E. Simova, V.R. Bhardwaj, D.M. Rayner, P.B. Corkum, "Polarizationselective etching in femtosecond laser-assisted microfluidic channel fabrication in fused silica", Optics Letters, 30, 14, (2005), 1867-1869

[7] D. Wortmann, J. Gottmann, N. Brandt, H. Horn-Solle, "Micro- and nanostructures inside sapphire by fs-laser irradiation and selective etching", Optics Express, 16, (2008), 1517-1522

[8] Y. Bellouard, A. Said, M. Dugan, P. Bado, "Fabrication of high-aspect ratio micro fluidic channels and tunnels using femtosecond laser pulses and chemical etching”, Optics Express, 12, (2004), 2120

[9] S. Matsuo, Y. Shickijo, T. Tomita, S. Hashimoto: "Laser fabrication of ship-in-a-bottle microstructures in sapphire", JLMN, 2, (2007), 114-116

[10] R. Osellame, H.J.W.M. Hoekstra, G. Cerullo, M. Pollnau, "Femtosecond laser microstructuring: an ena-bling tool for optofluidic lab-onchips",Laser Photonics Rev., 5, (2011), 442-463

[11] J. Gottmann, M. Hermans, J. Ortmann, "Microcutting and Hollow 3D Microstructures in Glasses by In-Volume Selective Laser-induced Etching (ISLE)", JLMN, 8, 15-18

[12] P.G. Kazansky, M. Beresna, Y. Shimotsuma, K. Hirao, Y. Svirko, "New phenomena in interaction of intense light pulses with transparent materials: from 3D self-assembled nanostructures to quill writing and nonreciprocal photosensitivity", Proc. of SPIE., Vol 7600, (2010), p.760017

[13] C. Corbari, A. Champion, M. Gecevicius, M. Beresna, Y. Bellouard, P.G. Kazansky, "Femtosecond versus picosecond laser machining of nano-gratings and micro-channels in silica glass", Optics Express, 21, 4, (2013), 3946-3958

[14] Y. Bellouard, M. Hongler, "Femtosecondlaser generation of self-organized bubble patterns in fused silica", Optics Express, 19, 7, (2011)

[15] W. Yang, E. Bricchi, P.G. Kazansky, J. Bovatsek, A.Y. Arai, "Self-assembled periodic subwavelength structures by femtosecond laser direct writing”, Optics Express, 14, 21, (2006), 10117-10124

[16] S. Richter, F. Jia, M. Heinrich, S. Döring, U. Peschel, A. Tünnermann, S. Nolte, „The role of selftrapped excitons and defects in the formation of nanogratings in fused silica“, Optics Letters, 37, 4, (2012)
[17] C. Hnatovsky, R.S. Taylor, E. Simova, P.P. Rajeev, D.M. Rayner, V.R. Bhardwaj, P.B. Corkum, "Pulse duration dependence of femtosecond-laserfabricated nanogratings in fused silica", Appl. Phys. Letters, 87, (2005), 014104

[18] C. Hnatovsky, R.S. Taylor, P.P. Rajeev, E. Simova, V.R. Bhardwaj, D.M. Rayner, P.B. Corkum, "Fabrication of microchannels in glass using focused femtosecond laser radiation and selective chemical etching", Appl. Phys. A, 84, (2006), 47-61

[19] J. Canning, M. Lancry, K. Cook, A. Weickman, F Brisset, B. Poumellec, "Anatomy of a femtosecond laser processed silica waveguide", Optical Materials Express, 1, 5, (2011), 998-1008

[20] H. Seidel, L. Csepregi, A. Heuberger, H. Baumgärtel, „Anisotropic Etching of Crystalline Silicon in Alkaline Solutions", J. Electrochem. Soc., 137, (1990), 11

(Received: August 19, 2013, Accepted: April 14, 2014) 